\title{
The Problem of Outward Cellular Electric Current
}

\author{
Mark Noble* \\ Department of Medicine and Therapeutics, UK
}

*Corresponding author: Mark Noble, Department of Medicine and Therapeutics, University of Aberdeen, UK.

Received Date: April 10, 2019

Published Date: April 25, 2019

\begin{abstract}
An outward electrical current from a living cell during repolarisation implies a flow of electrons in the opposite direction according to the definition of electricity (electrons moving). As there are no free electrons in the interstitial fluid that bathes the living cell membrane, an outward current is an impossibility unless an ATP driven exchanger is utilized. It is postulated that during repolarisation electrons flow from the high electron dense mitochondria into the general cell cytoplasm, thus restoring the cytoplasmic electric potential to its stable (inactive) value.
\end{abstract}

\section{Introduction}

The interstitial fluid constitutes the body's internal environment that bathes all of the living cells in the body. Its composition is therefore crucial for normal functions and is maintained by a number of homeostatic mechanisms involving negative feedback. Homeostasis regulates, among other factors, the $\mathrm{pH}$, sodium, potassium, and calcium concentrations in the extracellular fluid including the interstial fluid. The volume of body fluid, blood glucose, oxygen, and carbon dioxide levels are also tightly homeostatically maintained. The $\mathrm{pH}$ is determined by the hydrogen ion concentration, the hydrogen ion being positively charged electrically because it is a hydrogen atom with the electron missing, i.e., a proton. Any electrons, which have a negative electric charge, that are not part of atoms would combine with these protons to form hydrogen, a gas. Thus, there are no free electrons in interstitial fluid, only those within atoms and ions. The interstitial fluid is separated from the interiors of the cells by the cell membrane (also called plasma membrane, plasmalemma, or in the case of muscle cells sarcolemma), which is a double lipid layer (two lamellae) of lipoproteins, the lamellae being separated by an interlamellal space. The intracellular material which is in a gel form has a negative electric potential, i.e., is negatively charged, and therefore has electrical energy. This produces an electric field force across the cell membrane. The interior of the resting cells, e.g., neuron or muscle cell is at an electrical potential about $50-100$ $\mathrm{mV}$ more negative than the interstitial fluid. Although this potential difference seems small, it exists across a plasma membrane only about $5 \mathrm{~nm}$ thick, so that the resulting voltage gradient is about
$100,000 \mathrm{~V} / \mathrm{cm}$ [1]. Such an electric field force is sufficient to exclude all charged particles from the interlamellal space, so that no movement of charged particles (ions, protons and electrons) can flow across the membrane. This is the resting, or stable state of living cells.

\section{The Unstable State of Living Cells}

Many cells, when activated to perform a function, lose some or all of their negative charge. i.e., they depolarise. The voltage in the depolarised state is proportional to the electrons lost in the action. In the case of a livng cell, this is registered as current passing from the exterior into the cell, but a current is, by definition, a flow of electrons in the opposite direction. Electrons do not behave like classical physical objects; they can be in more than one position at the same time, can behave as an electromagnetic wave, and have also been described as a "cloud" by nuclear physicists. Nevertheless they have mass, albeit very small (9.10938356 × 10-31 kilograms). This is four orders of magnitude less than the mass of a proton $(1.6726219 \times 10-27$ kilograms) and is at least one 10 thousanth of the mass of any other ion. Thus if there is some influence in the interstitium that lowers the resistance of the cell membrane, electrons will accelerate from the high electron density of the intracellular gel 10,000 times faster than any ion, atom or molecule can enter the cell along its concentration gradient. The electrons have to pass through the lower resistance or impedance of the cell membrane, so the their energy is converted to heat.

An example of this is the pancreatic islet $ß$ cells which maintain glucose homeostasis by secreting insulin. The intracellular negative 
potential decreases and action potentials occur with increasing frequency as the glucose concentration increases above $4 \mathrm{mM}$ [2], i.e., the normal target extracellular concentration to be maintained. Action potentials are clustered in bursts that are interrupted by hyperpolarized interburst phases [3], i.e., episodes of increased electron loss interspersed by electron gain. Where do the electrons come from when there is a hyperpolarisation burst? The same authors [3] reviewed all the ion channels in islet cells and concluded that the depolarizing current of beta-cells occurred by an as yet unknown ion channel. However, all one has to postulate is that glucose increases the electrical conductance of the cell membrane leading to electron flow from the intracellular to the extracellular compartment. i.e., depolarisation. In most cells the only evidence for positive ion entry is the case of calcium ions $\left(\mathrm{Ca}^{2+}\right)$ which trigger many cellular functions. In the case of pancreatic islet cells, Dean and Matthews concluded that the action potentials induced by glucose are due predominantly to calcium entry and that sodium ions $\left(\mathrm{Na}^{+}\right)$tend to repress this calcium influx [4]. Most authors on this subject agree that the frequency of action potentials controls insulin secretion, so that, as with many other cell types, $\mathrm{Ca}^{2+}$ may be the trigger for function. However, $\mathrm{Ca}^{2+}$ entry must be strictly controlled and balanced by equal $\mathrm{Ca}^{2+}$ extrusion if cell death due to precipitation of the phosphate based energy generating function is to be prevented.

The slow depolarisation of pancreatic islet cells with rising extracellular glucose concentration, leading eventually to $\mathrm{Ca}^{2+}$ dependent action potentials would fit with the general hypothesis that the gradual loss of intracellular electrical negativity induces detachment of $\mathrm{Ca}^{2+}$ from its binding to the anionic phospholipids of the inner lamella of the cell membrane, such loss of binding opening the $\mathrm{Ca}^{2+}$ channels. A similar mechanism seems to apply to sinus node cells which show a steady depolarisation during diastole from about $-60 \mathrm{mV}$ to about $-40 \mathrm{mV}$ when a $\mathrm{Ca}^{2+}$ dependent action potential occurs that is transmitted to the rest of the heart and triggers the heartbeat. No ingoing electrically positive ion has been identified to cause the diastolic depolarisation, leading to the naming of this current as "funny current" (If) [5]. At the threshold potential near $-40 \mathrm{mV}$, the triggered action potential is $\mathrm{Ca}^{2+}$ dependent [6]; it is postulated that the field force across the cell membrane at -60 to $-40 \mathrm{mV}$ potential is insufficient to prevent detachment of $\mathrm{Ca}^{2+}$ from the anionic phospholipids of the inner lamella of the cell membrane which opens the $\mathrm{Ca}^{2+}$ channel [7] and leads to further outward flow of electrons and intracellular electron loss. The $\mathrm{Ca}^{2+}$ entry is matched by extrusion by the sodium/calcium exchanger NCX [8].

\section{Replacement of Electron Losses}

A characteristic of living cells that depolarise to trigger function is that the transmembrane potential recovers to an extent that varies with cell type. It is commonly assumed that this is achieved by an outward current carried by positively charged ions. This arose from the conventional idea of positive electric polarity, but we know that a positive current is actually a flow of electrons in the opposite direction implying a flow of electrons from the interstitium into the cell. But as explained in the introduction above, there cannot be any free electrons in the interstitial fluid; thus an outward current as conventionally measured cannot represent reality, i.e., a positive outward current is impossible. A more simple explanation of repolarisation (and hyperpolarisation bursts, e.g., in pancreatic islet cells) is re-supply of electrons to compensate for the electron loss during depolarisation. This seems highly likely when one considers that the electric potential of mitochondria is $-180 \mathrm{mV}$ [9].

Having just pointed out that outward currents are impossible, how can it be that outward currents are actually recorded during repolarisation? The best modern measurements by the Verkerk and Wilders group show that these are tiny currents in the picoamp range, the net current traces also being "noisy"; they have the same waveform shape as the first time derivative of the transmembrane potential recording. Therefore, this $\mathrm{dV} / \mathrm{dt}$ clean trace is used to indicate current rather than the actual net current, e.g., [10]. This gives a clue to an alternative explanation: - Suppose that the transmembrane potential indicates loss of electrons from the general cytoplasm during depolarisation and that during repolarisation, the general cytoplasm is being replenished by a flow of electrons from the high electron dense mitochondria. The apparent outward current registered by $\mathrm{dV} / \mathrm{dt}$ would actually be the rate of such replenishment and not an inward flow of electrons from interstitial fluid that would be required for a true outward current.

\section{True Currents That Do Cross the Plasmalemma}

The sodium/calcium exchanger (NCX) that expells $\mathrm{Ca}^{2+}$ equivalent to the $\mathrm{Ca}^{2+}$ that enters via the $\mathrm{Ca}^{2+}$ channel [8] creates a current. 3 sodium ions $\left(\mathrm{Na}^{+}\right)$enter for each $\mathrm{Ca}^{2+}$ expelled, creating an inward current which can be measured [10]. The sodium that enters raises intracellular $\mathrm{Na}^{+}$concentration is expelled by the sodium/potassium $\left(\mathrm{Na}^{+} / \mathrm{K}^{+} \mathrm{ATPase}\right)$ exchanger (sodium pump), with $3 \mathrm{Na}^{+}$expelled in exchange for $2 \mathrm{~K}^{+}$creating an outward current with loss of chemical energy. The sodium pump takes about 10 minutes to respond to an increase in activity [11]. $\mathrm{K}^{+}$outflow is slow, taking about 40 minutes [12].

There are a multiplicity of patch clamp studies in which electrical stimulation of a patch of cell membrane evokes $\mathrm{K}^{+}$dependent responses. The electrical energy in such studies is supplied by the experimental apparatus, so that it is difficult to assess the relevance of the results to an intact cell or organism. What seems to be lacking is a measurement of $\mathrm{K}^{+}$leaving a cell during rapid repolarisation [13] recorded fluctuations in extracellular $\mathrm{K}^{+}$during the sinus node cycle but were worried about the method used Kronhaus et al. If $\mathrm{K}+$ did leave cells during repolarisation, the cell would become depleted of $\mathrm{K}^{+}$because the defecit cannot be made up by the sodium pump [14]. In any case the amount of postulated $\mathrm{K}^{+}$outflow is insufficient to achieve repolarisation [15].

\section{Conclusion}

It is postulated that repolarisation of cells after depolarisation occurs by a mechanism of electron flow from mitochondria to the general cell cytoplasm, and not by an outward positive current.

\section{Acknowledgement}

None. 


\section{Conflict of Interest}

No conflict of interest.

\section{References}

1. Alberts B, Johnson A, Lewis J (2002) Ion Channels and the Electrical Properties of Membranes. Molecular Biology of the Cell New York: Garland Science 4th edition.

2. Dean PM, Matthews K (1970) Glucose-induced electrical activity in pancreatic islet cells. J. Physiol 210: 255-264.

3. Drews G, Krippeit Drews P, Düfer M (2010) Electrophysiology of islet cells. Adv Exp Med Biol 654: 115-163.

4. Dean PM, Matthews K (1970) Electrical activity in pancreatic islet cells: effect of ions. J Physiol 210: 265-275.

5. Di Francesco D (2010) The role of the funny current in pacemaker activity. Circ Res 106(3): 434-46.

6. Hagawara N, Irisawa H, Kameyama M (1988) Contribution of two types of calcium currents to the pacemaker potentials of rabbit sino-atrial node cells. J Physiol 395: 233-253.

7. Noble MIM (2018) On the Mechanism of the Cardiac L-type Calcium Channel in Cardiac Cells. Open J Cardiol Heart Dis 1(2): OJCHD.000507.

8. Eisner D, Bode E, Venetucci L, Trafford A (2012) Calcium flux balance in the heart. J Mol Cell Cardiol 58: 110-117.
9. Perry SW, Norman JP, Barbieri J, Brown EB, Gelbard HA, et al. (2011) Mitochondrial membrane potential probes and the proton gradient: a practical usage guide. Biotechniquies 50: 98-115.

10. Verkerk AO, Van Borren MMGJ, Wilders R (2013) Calcium transient and sodium- calcium exchange current in human versus rabbit sinoatrial node pacemaker cells. The Scientific World Journal Pp. 10.

11. Boyett MR, Hart G, Levi AJ, Roberts A (1987) Effects of repetitive activity on developed force and intracellular sodium in isolated sheep Purkinje fibres. J Physiol 388: 295-322.

12. Hodgkin AL, Keynes RD (1955) The potassium permeability of a giant nerve fibre J Physiol 128: 61-88.

13. Kronhaus KD, Spear JF, Moore EN, Kline RP (1978) Sinus node extracellular potassium transients following vagal stimulation. Nature 275: $322-324$

14. Ling GN (2007) History of the membrane (Pump) theory of the living cell from its beginning in mid-19th century to its disproof 45 years ago though still taught worldwide today as established truth. Physiol Chem Phys Med NMR 39: 1-67.

15. Noble MIM (2018) On the Mechanism of Depolarisation and Repolarisation in the Sino- Atrial Node. Open J Cardiol Heart Dis 1(2): OJCHD.000507. 